\title{
INVESTIGACIONES
}

\section{Promoviendo aprendizajes significativos en la enseñanza universitaria de la Historia a través de un juego de roles*}

\author{
Promoting significant learnings in the university teaching \\ of History through a role play
}

\section{Humberto Álvarez Sepúlveda}

\author{
${ }^{a}$ Departamento de Didáctica de la Facultad de Educación. \\ Universidad Católica de la Santísima Concepción, Chile. \\ halvarez@ucsc.cl
}

\begin{abstract}
RESUMEN
La presente investigación tiene como objetivo evaluar un juego de roles implementado en un grupo de estudiantes de Pedagogía en Educación Básica, quienes tuvieron que interpretar a un personaje destacado de la historia chilena. Es una investigación exploratoria de corte cualitativo basada en un estudio de casos y en un diseño transeccional. Se utilizó la observación participante de las clases donde se realizó la experiencia, por lo que los instrumentos consignados para la recogida de datos fueron el cuaderno de campo diseñado por los estudiantes, el diario del investigador, la planilla de asistencia y la rúbrica analítica elaborada para evaluar la actividad. Los resultados obtenidos muestran una mejora en el aprendizaje del alumnado; no obstante, también se constataron varias dificultades en la propuesta, tales como la escasa familiarización de los futuros docentes con la estrategia y la gran carga de trabajo que implicó para el docente y el estudiantado.
\end{abstract}

Palabras claves: innovación pedagógica, formación inicial docente, Historia de Chile, pensamiento histórico.

\begin{abstract}
This research aims to evaluate a role play implemented in a group of students of Basic Education Pedagogy, who had to play a prominent character in Chilean history. It is a qualitative exploratory research based on a case study and a transectional design. Participant observation of the classes where the experience was carried out was used, so the instruments consigned for data collection were the field notebook designed by the students, the researcher's diary, the attendance sheet and the analytical rubric prepared to evaluate the activity. The results obtained show an improvement in student learning; however, several difficulties were also noted in the proposal, such as the poor familiarization of future teachers with the strategy and the great workload it implied for teachers and students.
\end{abstract}

Key words: pedagogical innovation, initial teacher education, History of Chile, historical thinking.

\footnotetext{
* Este trabajo se enmarca en el Proyecto FAD 13/2019 "El debate y el juego de roles como metodologías activas en la enseñanza de la Historia. Una experiencia en Educación Superior”, adscrito a la Universidad Católica de la Santísima Concepción, Chile. Se agradece a la institución patrocinante por el apoyo otorgado.
} 


\section{INTRODUCCIÓN}

El desarrollo de competencias asociadas a la enseñanza y evaluación de la Historia es una parte fundamental de la formación inicial del profesorado de Educación Básica, porque a nivel nacional se trata de una carrera que se ha visto en la obligación de evolucionar a la par con los cambios que suceden en el sistema educacional y en el mercado laboral. Entre estos, destaca la necesidad de reorientar la enseñanza y la evaluación de la disciplina hacia enfoques pedagógicos más constructivistas y cercanos al mundo profesional, puesto que las universidades se ven cada vez más inmersas en la creciente globalización del conocimiento (López, 2020; Van Boxtel, Van Drie y Stoel, 2020).

A pesar de lo anterior, y como señala Álvarez (2020), prevalece una gran resistencia por parte de los académicos, quienes, de forma habitual, se muestran reticentes al momento de implementar metodologías didácticas y evaluativas innovadoras. No obstante, en la última década, se ha venido gestando una clase de Historia que combina la lección magistral y sus evaluaciones, basadas en la memorización de fechas, personajes y acontecimientos, con una serie de estrategias didácticas y evaluativas más modernas, centradas en el desarrollo del pensamiento histórico y de habilidades blandas por parte del alumnado. La adopción de dicho enfoque es un importante avance en el campo de la docencia universitaria, pero no es una propuesta definida y sólida para promover una clase de Historia innovadora y coherente con las nuevas necesidades formativas que requieren las futuras generaciones de estudiantes, para que puedan convertirse en ciudadanos responsables y críticos capaces de solucionar los conflictos en forma pacífica.

En este escenario, la mayor implementación de estrategias encaminadas al fomento de aprendizajes significativos resulta fundamental para que los docentes en formación inicial puedan potenciar su desempeño académico de forma integral, cuya condición es vital para que, al graduarse, estén altamente calificados para formar a los ciudadanos y ciudadanas del tercer milenio. Para lograr dicho objetivo, es importante que las estrategias didácticas y evaluativas utilizadas en el aula motiven el desarrollo de procesos de enseñanza-aprendizaje donde el estudiante sea un artífice importante de su propia formación, dejando al docente sólo como un tutor o guía de dicho proceso (Itzkovich, 2019; Álvarez, 2020).

Dentro de las metodologías más significativas e innovadoras, destaca el juego de roles porque fomenta el trabajo colaborativo, la confianza de los estudiantes y un nivel más profundo en las habilidades de comunicación oral, beneficios que avalan de manera consistente el uso de estrategias en donde el alumno adquiera un mayor protagonismo con su aprendizaje y tenga más interacción con sus compañeros de clase (Carbó y Pérez, 2010; Beidatsch y Broomhall, 2010; Fernández, Prieto, Alcaraz, Sánchez y Grimaldi, 2018).

En el contexto descrito, el presente artículo tiene como objetivo evaluar una experiencia de innovación basada en un juego de roles implementado en las asignaturas de "Ciencias Sociales II" y "Conociendo Chile de los siglos XIX y XX" de la carrera de Pedagogía en Educación Básica de una universidad del sur de Chile. Esta intervención se realiza bajo la convicción de que puede lograr que los profesores en formación desarrollen aprendizajes significativos (Ausubel, 1976) y alcancen a progresar en base a su esfuerzo por la "zona de desarrollo próximo" (Vygotsky, 1989) para comprender no solo los procesos históricos de manera lúdica y rigurosa, sino también para reconocer y reflexionar sobre las fortalezas y debilidades del juego de roles como estrategia didáctica y evaluativa. 


\section{EL JUEGO DE ROL EN LA EDUCACIÓN SUPERIOR}

Como destaca Young y Shaw (2014), el contexto universitario es complejo y sistémico, ya que involucra una serie de elementos materiales (infraestructura, biblioteca, recursos financieros, etc.) y humanos (un adecuado cuerpo académico, un conjunto de principios institucionales, entre otros) que son necesarios para que el estudiante pueda desarrollar sus competencias y desempeñar actividades específicas vinculadas a su futura labor. En este ámbito, la enseñanza universitaria se presenta como el eje central en el proceso de capacitación y actualización académica del alumnado, cuyo andamiaje es el proceso de enseñanza-aprendizaje a través del cual se promueven, se dirigen, se conducen o se evalúan aprendizajes significativos y acreditables (Monereo y Domínguez, 2014).

Sin embargo, la docencia universitaria, aunque recientemente se está acercando a paradigmas más constructivistas, se sustenta principalmente en enfoques pedagógicos tradicionales, en donde se aprecia, entre otros aspectos, la supremacía de la clase magistral, el dominio de la teoría por sobre la práctica y la prevalencia de evaluaciones centradas mayormente en la repetición y memorización de contenidos (Gaete, 2011; Gómez, Rodríguez y Mirete, 2018). Estos factores, sin duda, condicionan la adecuada formación académica de los y las estudiantes.

Adicionalmente, como precisa Gaete (2011), hay que considerar que, en muchas ocasiones, el análisis y la evaluación de los contenidos en la universidad se realiza en total desconexión con la estructura cognitiva previa que posee el alumno, por lo que se ignora la posibilidad de desarrollar un aprendizaje significativo ligado a esta. Para sortear este problema, es indispensable que los académicos estén dispuestos a utilizar estrategias innovadoras, o a lo menos diferentes a las convencionales. Dentro de estas metodologías, el juego de roles es, sin duda, una opción eficaz para promover y evaluar aprendizajes significativos en el alumnado. Como indica Craciun (2010), dicha estrategia nació del psicodrama, pero posteriormente fue aplicada a otras áreas educativas con la finalidad de ayudar a los y las estudiantes a comprender diversos aspectos complejos de la literatura y de las ciencias sociales y exactas.

Desde un punto de vista conceptual, y siguiendo a Murphy (2011), se trata de un juego en el que, tal como indica su nombre, uno o más jugadores desempeñan un determinado rol o papel. Cada jugador (estudiante) interpreta a un personaje único y diferente, con personalidad y características distintas, dependiendo del propósito individual, grupal o de los requisitos del profesor al momento de asignar la tarea. El objetivo del juego de rol es que todos los jugadores persigan un fin común, por lo que deben establecer un trabajo colaborativo para lograrlo. De esta manera, los roles desempeñados por los miembros del equipo permiten asumir una determinada responsabilidad según el grado de participación en la toma de decisiones relativas a la actividad.

El juego de rol posee una creciente demanda por parte de docentes y estudiantes, especialmente por la posibilidad que ofrece para combinar o complementar nueva información con los conocimientos previamente adquiridos (Suárez, Castro y Muñoz, 2020). Su vertiginoso protagonismo se explica también porque es una técnica capaz de promover la participación de todos los alumnos y crear situaciones en que se rompen los convencionalismos sociales, lo que favorece el desarrollo de habilidades interpersonales como la comunicación, el liderazgo, la responsabilidad y el trabajo colaborativo (Wenner, 2011; Quezada, Castro, Oliva, Gallo y Quezada, 2020). 


\section{EL JUEGO DE ROL COMO ESTRATEGIA DIDÁCTICA Y EVALUATIVA EN LA EDUCACIÓN HISTÓRICA UNIVERSITARIA}

En el marco de la formación inicial del profesorado de Educación Básica, el juego de roles, a diferencia de las teatralizaciones que se realizan en el aula para despertar la motivación del estudiantado, puede desempeñar también un papel fundamental en el desarrollo de aprendizajes pedagógicos e históricos significativos. De acuerdo con lo que plantean Gómez, Miralles, López y Prats (2017), esta estrategia dentro de la enseñanza y evaluación de la especialidad se concibe como una técnica de indagación, que tiene como principal objetivo que los alumnos y las alumnas investiguen e interpreten a un personaje o situación histórica específica, atendiendo a las características y a la complejidad de los hechos representados. Por esta razón, el juego de rol es una de las metodologías más eficaces para entender el pasado. Según Grande de Prado y Abella (2010), el pasado es difícil de comprender si el alumno no lo "reproduce" o no se "acerca" todo lo posible a él, puesto que no entenderá en ningún momento un acontecimiento o figura histórica hasta que no forme parte de su contexto. En este sentido, esta estrategia ofrece la posibilidad de "reconstruir" el pasado a partir de una actividad de simulación.

Con el juego de roles no solo se pretende que los y las estudiantes indaguen, observen, interactúen, representen y reflexionen acerca de la Historia, sino que también a través de la empatía logren borrar los prejuicios que surgen hacia los sucesos del pasado, tales como la supuesta imposibilidad de conocerlos de forma "rigurosa" y la escasa utilidad social de "reconstruirlos". Por tal motivo, es fundamental que el alumnado, mediante la interpretación de un determinado personaje y de su respectivo contexto, pueda reflexionar sobre su forma de pensar y actuar, y comprender sus posibles motivaciones de vida.

Dentro de esta dinámica, el docente necesariamente debe impulsar un proceso de enseñanza-aprendizaje que involucre conceptos, procedimientos y actitudes. Esta acción evita convertir el juego de roles en un simple ejercicio memorístico, que entraría en contradicción con esta metodología innovadora, ya que su propósito es promover aprendizajes significativos y potenciar el trabajo colaborativo del estudiantado para completar una tarea. Por ejemplo, el juego de roles como sustento de una actividad enmarcada en la clase de Historia puede requerir que los futuros docentes investiguen sobre un personaje y luego lo representen para entender sus valores y vivencias. En esta línea, el alumnado puede interpretar a su personaje a través de una entrevista simulada, una personificación, una dramatización u otro formato que estime conveniente.

Por su carácter lúdico, esta estrategia tiene múltiples motivaciones para el profesorado en formación, entre las cuales se destacan: la posibilidad de asumir ideas y posiciones distintas a las propias, el aprendizaje colaborativo, el empoderamiento en la toma de decisiones dentro del juego y el mayor compromiso con la asistencia a clases. Estas razones, siguiendo a Gómez, Ortuño y Miralles (2018), son suficientes para que el cuerpo académico considere la posibilidad de incorporar en el aula este tipo de metodologías.

La aplicación del juego de rol en el proceso de enseñanza-aprendizaje de la Historia puede ayudar a complementar y/o disminuir el predominio de las clases magistrales y las evaluaciones tradicionales en el aula, pues es una estrategia capaz de promover la formación del pensamiento histórico en los futuros docentes. Siguiendo dicho enfoque, diversos autores han defendido una enseñanza y una evaluación focalizada en el pensamiento histórico y no en la simple acumulación de información sobre los grandes acontecimientos 
que influyeron en Occidente o en los personajes más destacados de las historias nacionales (Gómez y Chapman, 2017; Molina y Ortuño, 2018; Romera, López y Monteagudo, 2019).

De acuerdo a Seixas y Morton (2012), el pensamiento histórico puede definirse como el proceso creativo que realizan los historiadores para interpretar fuentes del pasado y generar sus narrativas históricas. Siguiendo a Gómez, Miralles y Chapman (2017), dirigir el proceso educativo del alumnado hacia la formación del pensamiento histórico exige un cambio en la enseñanza y la práctica en el aula, que conlleva también una reflexión sobre la naturaleza de la evaluación del estudiantado y las estrategias utilizadas para la misma, ya que implica trascender del proceso evaluativo sumativo al formativo. De esta manera, la enseñanza y evaluación son dos procesos que se entrelazan e intercambian en el proceso de aprendizaje de los futuros profesores. Al respecto, Palacios, Chaves y Martin (2020) señalan que tanto en las actividades de aprendizaje como en los procesos de evaluación de la clase de Historia resulta pertinente incorporar y desarrollar aprendizajes que vayan más allá de conocimientos declarativos de primer orden (el qué, dónde, cuándo o quién ha llevado a cabo distintas acciones en un relato cronológico), con el fin de privilegiar procedimientos y aprendizajes de segundo orden del pensamiento histórico. Para Gómez, Ortuño y Molina (2014)

Estos se definen por la posesión o despliegue de diferentes estrategias, capacidades o competencias para responder a cuestiones históricas y entender de una forma más compleja el pasado. Este tipo de conocimientos históricos están relacionados con habilidades propias del historiador, que se concretan en la búsqueda, selección y tratamiento de fuentes (p. 10).

El reto, por tanto, es conseguir organizar un proceso didáctico-evaluativo de la Historia en el que se considere los fundamentos de la disciplina y los contenidos procedimentales propios del historiador. Esto, por ejemplo, se puede materializar en el trabajo directo con fuentes y en el posterior análisis de diferentes interpretaciones existentes sobre un determinado proceso histórico (Chapman, 2011). De este modo, el profesor puede contribuir a dotar al alumnado de una serie de conocimientos, procedimientos y actitudes que le permitan abordar el estudio de la disciplina con autonomía para construir su propia representación del pasado, y así pueda ser capaz de contextualizar o juzgar los hechos históricos, consciente de la distancia que los separa del presente (Prats, 2016; Gómez, Ortuño y Miralles, 2018).

Esto último contribuye indudablemente a la formación del pensamiento histórico, cuyo metaconcepto debe estar al servicio de la comprensión del mundo actual para que los alumnos y las alumnas puedan forjar una ciudadanía democrática que les permita gestionar de mejor manera el cambio (Domínguez, 2015; Gómez y Miralles, 2015; Armas, Moreira, Maia y Conde, 2019). En esta línea, es necesario e imprescindible que los futuros docentes posean una adecuada formación en la especialidad para evitar la idea de invalidez y de inutilidad del conocimiento histórico (Gómez, Ortuño y Molina, 2014).

En relación al juego de rol, cabe destacar que esta estrategia didáctica-evaluativa permite trabajar y articular simultáneamente los cinco conceptos fundamentales del pensamiento histórico; a saber, estos son: fuentes históricas, causas y consecuencias, tiempo histórico, relevancia histórica y empatía histórica (Gómez et al., 2014; Gómez, López y Prats, 2017). En el siguiente cuadro se presentan los conceptos citados con una breve definición y las competencias cognitivas que movilizan dentro de una actividad de juego de roles. 
Tabla 1. Cuadro de relaciones entre los conceptos del pensamiento histórico y las competencias cognitivas que se movilizan en el juego de roles

\begin{tabular}{|c|c|c|}
\hline Concepto & $\begin{array}{l}\text { Importancia que tiene dentro } \\
\text { del juego de roles }\end{array}$ & Competencias cognitivas que moviliza \\
\hline $\begin{array}{l}\text { Fuentes } \\
\text { históricas }\end{array}$ & $\begin{array}{l}\text { El análisis de fuentes históri- } \\
\text { cas es un procedimiento des- } \\
\text { criptivo y heurístico clave para } \\
\text { investigar sobre el rol (ligado } \\
\text { mayormente a un personaje) y } \\
\text { su respectivo contexto históri- } \\
\text { co, para poder representar la } \\
\text { temática asignada. }\end{array}$ & $\begin{array}{l}\text { - Leer y decodificar fuentes escritas, orales, icono- } \\
\text { gráficas, cartográficas, audiovisuales, materiales, } \\
\text { gráficas y estadísticas. } \\
\text { - Obtener información explícita e implícita por infe- } \\
\text { rencia. } \\
\text { Analizar y contrastar las distintas fuentes para in- } \\
\text { terpretar y evaluar la información de la temática en } \\
\text { su contexto. } \\
\text { - Asumir la existencia de distintas interpretaciones } \\
\text { historiográficas como un rasgo distintivo del cono- } \\
\text { cimiento histórico. }\end{array}$ \\
\hline $\begin{array}{l}\text { Causas y } \\
\text { consecuencias }\end{array}$ & $\begin{array}{l}\text { Este concepto ayuda al estu- } \\
\text { diante a entender la compleji- } \\
\text { dad del personaje o situación } \\
\text { representada, porque permite } \\
\text { analizar el pasado como una } \\
\text { dimensión que involucra di- } \\
\text { versos hechos y procesos don- } \\
\text { de intervienen factores multi- } \\
\text { causales y relacionales, que } \\
\text { desencadenan consecuencias } \\
\text { de distinta naturaleza. }\end{array}$ & $\begin{array}{l}\text { - Identificar y clasificar las causas de los fenómenos } \\
\text { de forma descriptiva y explicativa (contenido, fun- } \\
\text { ción, duración, entre otras). } \\
\text { - Analizar e interrelacionar causas-consecuencias. } \\
\text { - Formular hipótesis y emitir razonamientos contra- } \\
\text { fácticos. }\end{array}$ \\
\hline $\begin{array}{l}\text { Tiempo } \\
\text { histórico }\end{array}$ & $\begin{array}{l}\text { El tiempo histórico ayuda a } \\
\text { los estudiantes a situarse den- } \\
\text { tro de los procesos de cambio } \\
\text { y de continuidad, que inciden } \\
\text { sobre la vida del personaje o la } \\
\text { situación representada en el } \\
\text { juego de roles. }\end{array}$ & $\begin{array}{l}\text { Comprender y utilizar convenciones temporales } \\
\text { como década, siglo, milenio, antes y después de } \\
\text { Cristo, generación, entre otras. } \\
\text { Aplicar de forma flexible y creativa las variables } \\
\text { del tiempo histórico, como sucesión, simultanei- } \\
\text { dad, duración, entre otras. } \\
\text { Identificar y comparar las fases del tiempo históri- } \\
\text { co, tales como períodos de auge y crisis, cambio y } \\
\text { continuidad, entre otras. } \\
\text { Evaluar las dimensiones pasado, presente y futuro } \\
\text { de la experiencia histórica, para comprender la } \\
\text { complejidad y singularidad del hecho investigado. }\end{array}$ \\
\hline $\begin{array}{l}\text { Relevancia } \\
\text { histórica }\end{array}$ & $\begin{array}{l}\text { A partir de la relevancia his- } \\
\text { tórica que tienen en el pre- } \\
\text { sente, los estudiantes pue- } \\
\text { den discernir sobre aquellos } \\
\text { personajes o procesos histó- } \\
\text { ricos que resultan interesan- } \\
\text { tes y significativos para tra- } \\
\text { bajar. }\end{array}$ & $\begin{array}{l}\text { Seleccionar hechos y procesos que son necesa- } \\
\text { rios y pertinentes de estudiar para entender el } \\
\text { presente en perspectiva histórica. } \\
\text { Comparar pasado-presente y formular interro- } \\
\text { gantes sobre diversas problemáticas actuales. } \\
\text { Comprender la durabilidad, la perspectiva, la } \\
\text { profundidad y el impacto de las consecuencias } \\
\text { de fenómenos del pasado en el presente. }\end{array}$ \\
\hline
\end{tabular}




\begin{tabular}{|l|l|ll|}
\hline $\begin{array}{l}\text { Empatía } \\
\text { histórica }\end{array}$ & $\begin{array}{l}\text { Este concepto ayuda al estu- } \\
\text { diante a ponerse en el "lu- } \\
\text { gar" del personaje o situa- } \\
\text { ción histórica interpretada, } \\
\text { lo que evita, en cierta medi- } \\
\text { da, analizar la temática des- } \\
\text { de una perspectiva presen- } \\
\text { tista. }\end{array}$ & $\begin{array}{l}\text { Evitar el uso del presentismo en la comprensión } \\
\text { de la problemática investigada. }\end{array}$ \\
$\begin{array}{l}\text { Eludir la asimilación de juicios éticos infunda- } \\
\text { dos sobre personajes o hechos del pasado. }\end{array}$ \\
\hline
\end{tabular}

Fuente. Elaboración propia.

Para desarrollar estas competencias históricas, resulta imprescindible que el docente sea capaz de convertir una parte de las actividades didácticas en un proceso de aprendizaje por descubrimiento, donde el estudiante en vez de recibir los contenidos de forma pasiva pueda descubrir los conceptos y sus relaciones para integrarlos a su esquema cognitivo (Tarr, 2018). Por tal motivo, al momento de implementar metodologías innovadoras y constructivistas en la clase de Historia, es de vital importancia responder a la especificidades epistemológicas, pedagógicas y cognitivas de la disciplina, puesto que con estas no se busca analizar la capacidad de los alumnos y las alumnas para acumular o memorizar aprendizajes de primer orden como fechas, hitos y personajes, sino que se pretende promover y evaluar el desarrollo de habilidades blandas y de aprendizajes de segundo orden del pensamiento histórico. Para adoptar este principio, es necesario que los profesores de la especialidad posean una teoría sólida sobre el pensamiento histórico, el aprendizaje de la disciplina y la búsqueda de marcadores de progresión cognitiva.

\section{ANTECEDENTES EMPÍRICOS}

Existen numerosos estudios relacionados con el juego de rol y su uso didáctico y evaluativo en el aula universitaria de Historia. Según Gómez, Rodríguez y Mirete (2018), los y las estudiantes tienden a aburrirse en esta asignatura por considerarla memorística y poco útil. Sin embargo, la creciente utilización del juego de roles en la enseñanza y evaluación de la Historia evidencia su importancia como una estrategia que ayuda al alumnado a aprender la asignatura de forma lúdica y entretenida, permitiéndoles también comprender su utilidad en la vida cotidiana.

En este contexto, Carbó y Pérez (2010) realizaron un estudio sobre la efectividad del juego de rol para evaluar el grado de comprensión del conocimiento histórico alcanzado por varios participantes, entre los que se contaron varios doctores, licenciados y alumnos de diferentes facultades de la Universidad de Salamanca. Entre los hallazgos más relevantes, los investigadores descubrieron que los estudiantes aceptaron las actividades de juego de rol con entusiasmo, ya que desafiaban su creatividad y habilidad para pensar críticamente. Asimismo, evidenciaron una mejora significativa en sus conocimientos conceptuales. También pudieron constatar cambios procedimentales y actitudinales muy positivos al momento de emprender la búsqueda y consulta de fuentes para caracterizar e interpretar a sus personajes de forma contextualizada. 
Otro estudio importante es el de Cedric Beidatsch y Susan Broomhall (2010). A partir de una serie de investigaciones que exploraban el uso didáctico y evaluativo del juego de rol en la clase de Historia dentro de una carrera de pregrado, Beidatsch y Broomhall concluyeron que esta estrategia permite el desarrollo de experiencias de aprendizajes que ayudan a fortalecer una gama de habilidades personales y sociales en los estudiantes, así como también facilita la comprensión del contenido de la asignatura y el sentido de la práctica historiográfica por parte de estos.

\section{METODOLOGÍA}

\subsection{PARTICIPANTES}

La población de este estudio está compuesta por todos los alumnos y las alumnas de segundo a quinto año de la carrera de Pedagogía en Educación Básica de una universidad del sur de Chile, ya que en dichos niveles se imparten actividades curriculares relacionadas con la disciplina histórica y su respectiva enseñabilidad. En esta línea, es importante destacar que los primeros seis semestres de la malla curricular de esta carrera se enmarcan dentro de un plan de formación general, que comprende el ámbito pedagógico y las áreas disciplinares del currículum de Enseñanza Básica, y los tres últimos semestres engloban las asignaturas relacionadas con las especialidades y didácticas que involucran las Menciones de "Matemática y Ciencias Naturales" y de "Lenguaje, Comunicación y Ciencias Sociales".

La técnica de muestreo aplicada en dicha población fue de carácter no probabilístico por conveniencia (Gómez, 2006) y no estratificado (Hernández, Fernández y Baptista, 2014), puesto que, en primer lugar, el estudio necesitó de estudiantes que hayan cursado asignaturas de la carrera donde se impartan contenidos vinculados a la Historia y su didáctica; y en segundo lugar, requirió de un curso de la Mención en "Matemática y Ciencias Naturales" y otro de la Mención en "Lenguaje, Comunicación y Ciencias Sociales" de la carrera mencionada para trabajar con estudiantes de ambos perfiles formativos. Siguiendo este último criterio, cabe destacar que las asignaturas seleccionadas fueron "Ciencias Sociales II", que pertenece al primer segmento, y "Conociendo Chile de los siglos XIX y XX", al segundo. Así, la selección total de la muestra dependió del investigador, y no de una probabilidad aleatoria de elección, respondiendo a criterios selectivos intencionales y a las posibilidades de acceso a la misma, en función del grado de adecuación a los objetivos de estudio (Sabino, 2014).

Como se constata en la tabla 2, los sujetos participantes $(\mathrm{p}=27)$ son estudiantes de las asignaturas "Ciencias Sociales II" ( $\mathrm{p}=9$ ) y "Conociendo Chile de los siglos XIX y XX" $(\mathrm{p}=18)$. La muestra se compone de 24 alumnas $(88,9 \%)$ y 3 alumnos $(11,1 \%)$. La edad mínima de los participantes es de 21 y la máxima es de 54, con una media de 24 años. 
Tabla 2. Información de los sujetos participantes según asignaturas incluidas en la experiencia

\begin{tabular}{|l|l|l|c|c|c|}
\hline $\begin{array}{l}\text { Nombre de la } \\
\text { actividad curricular }\end{array}$ & Carrera & Semestre/año & $\begin{array}{l}\text { Cantidad de } \\
\text { alumnos }\end{array}$ & Hombres & Mujeres \\
\hline $\begin{array}{l}\text { Ciencias Sociales } \\
\text { II }\end{array}$ & $\begin{array}{l}\text { Pedagogía en } \\
\text { Educación Básica, } \\
\text { Mención en } \\
\text { Matemática y } \\
\text { Ciencias Naturales }\end{array}$ & Primer semestre/2019 & 9 & 1 & 8 \\
\hline $\begin{array}{l}\text { Conociendo Chile } \\
\text { de los siglos XIX } \\
\text { y XX }\end{array}$ & $\begin{array}{l}\text { Pedagogía en } \\
\text { Educación } \\
\text { Básica, Mención } \\
\text { en Lenguaje, } \\
\text { Comunicación y } \\
\text { Ciencias Sociales }\end{array}$ & Primer semestre/2019 & 18 & 2 & 16 \\
\hline
\end{tabular}

Fuente. Elaboración propia.

\subsection{CONTEXTO EDUCATIVO DE LA EXPERIENCIA}

Las asignaturas "Ciencias Sociales II" y "Conociendo Chile de los siglos XIX y XX" tienen un carácter teórico-práctico, que se traduce en el desarrollo de talleres y actividades de aprendizaje que buscan promover la formación del pensamiento histórico en el alumnado.

El programa de "Ciencias Sociales II" consta de siete unidades temáticas que se imparten durante el primer semestre, el cual tiene una duración de 18 semanas. Los principales ejes temáticos de las unidades mencionadas son: La llegada de los europeos a América, Colonia de Chile, Chile de los siglos XIX y XX, Zonas geográficas de Chile, Participación ciudadana y derechos civiles, Problemáticas ambientales e Importancia de los medios de comunicación y sus tipologías.

Por su parte, el programa de "Conociendo Chile de los siglos XIX y XX" contiene cuatro unidades temáticas que se trabajan durante el primer semestre. Los principales ejes temáticos de estas unidades son: Independencia de Chile (1810-1823), Organización de la República (1823-1830), República Conservadora (1831-1861), República Liberal (1861-1891), Ocupación de la Araucanía y Campaña del Desierto, Gobierno de la Unidad Popular (1970-1973), Dictadura de Augusto Pinochet (1973-1990) y Recuperación de la democracia.

En estas actividades curriculares, y a efectos de esta investigación, el juego de roles se presenta como una estrategia para desarrollar y evaluar aprendizajes significativos en el marco de la segunda unidad de "Ciencias Sociales II", que aborda la Colonia de Chile (1598-1810); y en el contexto de la primera unidad de "Conociendo Chile de los siglos XIX y XX”, que analiza la Independencia de Chile (1810-1823). Siguiendo esta distribución, los estudiantes, reunidos en equipos, tuvieron que representar a un personaje histórico según la unidad especificada para cada curso.

Los aspectos considerados para elaborar la actividad tienen relación con el conocimiento disciplinar de los periodos mencionados de la Historia de Chile y con las competencias cognitivas del pensamiento histórico, el cual se presenta como uno de 
los principales lineamientos de la didáctica de la especialidad y constituye una de las cualidades fundamentales que debe poseer el Profesor de Educación Básica. Por tal motivo, los contenidos mínimos que se consideraron para cada asignatura fueron:

\section{Solo aplica para "Ciencias Sociales II"}

- Esclavitud, merced y encomienda

- Sociedad del Chile colonial

- Personajes históricos icónicos de la Colonia

- Características culturales del Chile colonial

$\underline{\text { Solo aplica para "Conociendo Chile de los siglos XIX y XX" }}$

- Factores internos y externos que motivaron la independencia de Chile

- Personajes políticos y militares que lideraron el proceso de emancipación

- Batallas y conflictos bélicos de la independencia

\section{Aplica para ambas actividades curriculares}

- Enfoques didácticos en la enseñanza de la Historia

- El juego de rol: características y variantes

- Caracterización del pensamiento histórico

- Conceptos y competencias cognitivas del pensamiento histórico

- Cuaderno de campo: características, potencialidades y diseño

Los alumnos de cada asignatura recibieron los contenidos mencionados durante las tres semanas previas a la actividad. En el caso de "Ciencias Sociales II", esto se realizó en 12 horas totales (4 horas por semana); y en el de "Conociendo Chile de los siglos XIX y XX”, en 9 (3 horas por semana).

En este período, los y las estudiantes desarrollaron dos talleres de inducción al juego de roles, donde tuvieron que aplicar todos los conocimientos aprendidos en una representación de situaciones o hechos históricos de la Colonia (1598-1810) e Independencia de Chile (1810-1823). Asimismo, en dichos talleres, los futuros profesores implementaron un laboratorio histórico (Prats, 2011; Salazar, Orellana, Muñoz y Bellati, 2017; Álvarez, 2020), donde simularon el trabajo del historiador, a partir de la búsqueda, selección y tratamiento de fuentes, para preparar el montaje provisional o definitivo del juego de rol, incluyendo los diálogos, el vestuario y la escenografía requerida.

En cada taller, los alumnos contaron con retroalimentaciones regulares que les invitaban a mejorar sus propuestas mediante la reflexión crítica de sus fortalezas y debilidades.

Para desarrollar el juego de roles definitivo, el alumnado recibió el instructivo de trabajo consignado en la tabla 3. 
Tabla 3. Instructivo del juego de roles

1. Forme una pareja de trabajo, y utilizando la estrategia del juego de roles, prepare y desarrolle una representación de un personaje histórico de la Colonia o Independencia de Chile (dependiendo de la asignatura), considerando todos los elementos analizados y estudiados en clases para esta actividad. La propuesta debe tener una duración máxima de 15 minutos.

2. La asignación del personaje se realiza por sorteo, a partir de un listado de nombres confeccionado por el profesor. En el caso de "Conociendo Chile de los siglos XIX y XX", el listado está formado por los siguientes personajes: Bernardo O’Higgins, José de San Martin, Javiera Carrera, Manuel Rodríguez, Thomas Cochrane, José Miguel Carrera, Mateo de Toro y Zambrano, Paula Jaraquemada y Agueda Monasterio. Y, en el caso de "Ciencias Sociales II", el listado está formado por Ambrosio O’Higgins, Juan Ignacio Molina, Catalina de los Ríos y Lisperguer (La Quintrala) y Francisco Antonio García Carrasco.

3. Una vez asignado el personaje, implemente un laboratorio histórico para investigar y delimitar el aspecto de su vida que deseen representar. Para ello, debe regirse por el principio de las cuatro heurísticas propuestas por Salazar, Orellana, Muñoz y Bellati (2017). A saber, estas son:

- Heurística de origen, teniendo en cuenta de donde proviene el documento histórico y su propósito.

- Contextualización, con el fin de poner el documento en su contexto temporal y espacial.

- Lectura cerrada, para extraer la idea principal del documento.

- Corroboración heurística, para comparar las múltiples fuentes.

4. En el juego de roles, el protagonista es el personaje histórico representado. Sin embargo, tienen la posibilidad de desarrollar roles secundarios, lo cual dependerá de la creatividad y comprensión de la información biográfica de su personaje. Se sugiere construir un guion gráfico o storyboard para organizar la secuencia de las situaciones o hechos que se representaran sobre el personaje asignado.

5. El juego de roles debe ser presentado en un plazo máximo de una semana, que se cuenta a partir de la entrega del instructivo. En el caso de la asignatura "Ciencias Sociales II", los alumnos deben realizar su juego de rol el 3 de abril y los de "Conociendo Chile de los siglos XIX y XX” los días 3 y 5 de abril de 2019.

6. Cada equipo debe llevar el vestuario, utilería y escenografía que considere necesaria para caracterizar a su personaje, según el contexto histórico vivido por este. Este material debe ser confeccionado por los propios estudiantes, ya que no se aceptará el arriendo o compra de este. El objetivo de esta indicación es que puedan ser capaces de ponerse en el "lugar" del personaje a la hora de elaborar los insumos y, desde allí, tratar de entender el comportamiento y el entorno sociocultural del personaje a interpretar.

7. El formato de presentación del juego de rol queda a criterio de cada grupo. Puede ser una entrevista simulada, una personificación, una dramatización u otro formato que estimen pertinente.

8. Cada estudiante debe entregar un cuaderno de campo de 8 páginas donde conste una descripción de la actividad, la fecha de realización, el diseño de un esquema que explique la estrategia utilizada (dibujos, mapas mentales, mapas conceptuales, diagramas de flujo, entre otros) y la realización de una minuciosa reflexión sobre los desafíos y dificultades del juego de rol como estrategia didáctica y evaluativa dentro de la enseñanza de la Historia.

Fuente. Elaboración propia. 


\subsection{TÉCNICAS E INSTRUMENTOS DE RECOLECCIÓN DE INFORMACIÓN}

Como señala Evans (2010), es importante contar con técnicas e instrumentos de recolección confiables y rigurosos para cumplir con el propósito de la investigación. En efecto, se realizaron observaciones de clases para recoger información sobre la experiencia desde la perspectiva del alumno y del docente.

En cuanto a la óptica de los futuros profesores, se recurrió a los cuadernos de campo elaborados por ellos mismos para conocer su percepción sobre la estrategia implementada, abarcando aspectos tales como la descripción de la actividad, y las fortalezas y debilidades que tuvieron en el juego de rol en función de su desempeño.

Desde la postura docente, y en atención del objetivo propuesto, se realizó un proceso de observación participante para asegurar un proceso efectivo de retroalimentación que permitiese a los y las estudiantes mejorar sus aprendizajes. La observación se planteó desde una perspectiva amplia, ya que se intentó obtener una visión general de las sesiones. Para recabar datos de estas, se utilizó la planilla de asistencia, el diario del investigador y la rúbrica diseñada para evaluar el juego de roles.

La planilla de asistencia sirvió como insumo de información para constatar la concurrencia física de los estudiantes en las clases que contemplaron tanto los dos talleres de inducción al juego de rol como la ejecución del mismo. Cabe destacar que el porcentaje de asistencia fue un indicador relevante que permitió evidenciar el grado de responsabilidad y compromiso de los sujetos participantes con la experiencia de aula.

En cuanto al diario del investigador, y aunque la presente investigación se centra fundamentalmente en el alumnado, también es importante contar con la observación de la praxis por parte del docente porque permite mejorar considerablemente la calidad de la triangulación de los datos. Este instrumento posibilitó la recolección de datos relativos a los problemas y los beneficios que tuvieron los y las estudiantes durante la actividad, los aspectos que se deben considerar en futuras intervenciones y la valoración del alumnado sobre la propuesta. Para tales efectos, se tomaron notas puntuales en el diario del investigador y se grabaron mediante un dispositivo de voz las clases que consignaron el desarrollo íntegro del juego de roles.

Por último, la rúbrica (tabla 4) tuvo como propósito evaluar el desempeño académico evidenciado por los futuros docentes en los dos talleres de inducción y en la realización de la actividad, con el fin de constatar la evolución de su proceso de aprendizaje. En esta investigación se ha optado por diseñar y utilizar una rúbrica analítica (Gatica y Uribarren, 2013), pues permite desglosar la actividad en los seis aspectos consignados (laboratorio de historia, enfoque del tema, organización, vestuario y escenografía, tono de voz y desenvolvimiento). Cada uno de estos describen los criterios observables para cada nivel de logro, los cuales, en este instrumento particular, van de aprendiz a experto. Asimismo, este tipo de rúbrica es muy útil para analizar detalladamente cada una de las competencias asociadas al juego de roles, permitiendo detectar los puntos fuertes y débiles del individuo o la pareja en la ejecución del mismo. Finalmente, la rúbrica analítica posibilita un alto grado de retroalimentación profesor-alumno a la hora de establecer los criterios individuales de puntuación de los diferentes aspectos evaluados. 
Tabla 4. Rúbrica de evaluación del juego de roles

\begin{tabular}{|c|c|c|c|c|}
\hline Criterio & $\begin{array}{l}\text { Experto } \\
\text { (4 puntos) }\end{array}$ & $\begin{array}{l}\text { Avanzado } \\
\text { (3 puntos) }\end{array}$ & $\begin{array}{l}\text { Intermedio } \\
\text { ( } 2 \text { puntos })\end{array}$ & $\begin{array}{l}\text { Aprendiz } \\
\text { (1 punto) }\end{array}$ \\
\hline $\begin{array}{l}\text { Laboratorio de } \\
\text { historia }\end{array}$ & $\begin{array}{l}\text { En el laboratorio } \\
\text { histórico } \\
\text { implementado } \\
\text { para desarrollar } \\
\text { el juego de roles } \\
\text { se observa la } \\
\text { aplicación de las } \\
\text { cuatro heurísticas } \\
\text { (heurística } \\
\text { de origen, } \\
\text { contextualización, } \\
\text { lectura cerrada } \\
\text { y corroboración } \\
\text { heurística). }\end{array}$ & $\begin{array}{l}\text { En el laboratorio } \\
\text { histórico } \\
\text { implementado } \\
\text { para desarrollar } \\
\text { el juego de roles } \\
\text { no se observa } \\
\text { la aplicación } \\
\text { de una de las } \\
\text { cuatro heurísticas } \\
\text { (heurística } \\
\text { de origen, } \\
\text { contextualización, } \\
\text { lectura cerrada } \\
\text { y corroboración } \\
\text { heurística). }\end{array}$ & $\begin{array}{l}\text { En el laboratorio } \\
\text { histórico } \\
\text { implementado } \\
\text { para desarrollar } \\
\text { el juego de roles } \\
\text { no se observa } \\
\text { la aplicación de } \\
\text { dos o tres de las } \\
\text { cuatro heurísticas } \\
\text { (heurística } \\
\text { de origen, } \\
\text { contextualización, } \\
\text { lectura cerrada } \\
\text { y corroboración } \\
\text { heurística). }\end{array}$ & $\begin{array}{l}\text { En el laboratorio } \\
\text { histórico } \\
\text { implementado } \\
\text { para desarrollar } \\
\text { el juego de roles } \\
\text { no se observa } \\
\text { la aplicación de } \\
\text { ninguna de las } \\
\text { cuatro heurísticas } \\
\text { (heurística } \\
\text { de origen, } \\
\text { contextualización, } \\
\text { lectura cerrada } \\
\text { y corroboración } \\
\text { heurística). }\end{array}$ \\
\hline $\begin{array}{l}\text { Enfoque en el } \\
\text { tema asignado }\end{array}$ & \begin{tabular}{|l|} 
Entre el $90 \%$ \\
y el $100 \%$ del \\
juego de roles \\
presenta aspectos \\
biográficos del \\
personaje histórico \\
asignado de forma \\
entendible para \\
todo/el espectador.
\end{tabular} & \begin{tabular}{|l|} 
Entre el $60 \%$ \\
y el $89 \%$ del \\
juego de roles \\
presenta aspectos \\
biográficos del \\
personaje histórico \\
asignado de forma \\
entendible para \\
todo/el espectador.
\end{tabular} & $\begin{array}{l}\text { Entre el } 30 \% \text { y } \\
\text { el } 59 \% \text { del juego } \\
\text { de roles presenta } \\
\text { aspectos } \\
\text { biográficos } \\
\text { del personaje } \\
\text { histórico } \\
\text { asignado de } \\
\text { forma entendible } \\
\text { para todo/el } \\
\text { espectador. } \\
\end{array}$ & $\begin{array}{l}\text { Entre el 1\% } \\
\text { y el 29\% del } \\
\text { juego de roles } \\
\text { presenta aspectos } \\
\text { biográficos del } \\
\text { personaje histórico } \\
\text { asignado de forma } \\
\text { entendible para } \\
\text { todo/el espectador. }\end{array}$ \\
\hline Organización & \begin{tabular}{|l|} 
El juego de rol está \\
bien organizado. El \\
inicio, desarrollo \\
y cierre son \\
presentados de \\
forma muy clara.
\end{tabular} & $\begin{array}{l}\text { Una de las partes } \\
\text { del juego de rol } \\
\text { (inicio, desarrollo } \\
\text { o cierre) se } \\
\text { presenta de } \\
\text { manera confusa. }\end{array}$ & $\begin{array}{l}\text { Dos partes del } \\
\text { juego de rol } \\
\text { se presenta de } \\
\text { forma confusa. }\end{array}$ & $\begin{array}{l}\text { Las tres partes } \\
\text { del juego de rol } \\
\text { se presenta de } \\
\text { manera confusa, } \\
\text { por lo que resulta } \\
\text { muy complejo de } \\
\text { entender. }\end{array}$ \\
\hline $\begin{array}{l}\text { Vestuario y } \\
\text { Escenografía }\end{array}$ & $\begin{array}{l}\text { El vestuario y la } \\
\text { escenografía son } \\
\text { congruentes entre } \\
\text { sí y se enmarcan } \\
\text { totalmente en } \\
\text { la época del } \\
\text { personaje histórico } \\
\text { representado. }\end{array}$ & \begin{tabular}{|l} 
El vestuario y la \\
escenografía son \\
congruentes entre \\
sí, sin embargo, \\
presentan algunos \\
elementos que no \\
corresponden al \\
contexto histórico \\
del tema asignado.
\end{tabular} & $\begin{array}{l}\text { Existen varias } \\
\text { incongruencias } \\
\text { entre el } \\
\text { vestuario y la } \\
\text { escenografía, } \\
\text { y muchos de } \\
\text { sus elementos } \\
\text { no concuerdan } \\
\text { con la época del } \\
\text { tema asignado. }\end{array}$ & $\begin{array}{l}\text { El vestuario y la } \\
\text { escenografía son } \\
\text { completamente } \\
\text { incongruentes entre } \\
\text { sí y están totalmente } \\
\text { descontextualizados } \\
\text { del tema asignado, } \\
\text { o bien no presenta } \\
\text { la indumentaria } \\
\text { básica requerida } \\
\text { para representar al } \\
\text { personaje. }\end{array}$ \\
\hline
\end{tabular}




\begin{tabular}{|c|c|c|c|c|}
\hline Tono de voz & \begin{tabular}{|l|} 
El tono de \\
voz utilizado \\
hace posible la \\
comprensión de la \\
trama durante todo \\
el juego de roles.
\end{tabular} & $\begin{array}{l}\text { El tono de } \\
\text { voz utilizado } \\
\text { hace posible la } \\
\text { comprensión de la } \\
\text { narración durante } \\
\text { la mayor parte del } \\
\text { juego de roles. }\end{array}$ & $\begin{array}{l}\text { El tono de voz } \\
\text { dificulta la } \\
\text { comprensión de } \\
\text { la mitad de la } \\
\text { trama }\end{array}$ & $\begin{array}{l}\text { El tono de voz } \\
\text { dificulta la } \\
\text { comprensión de la } \\
\text { trama durante todo } \\
\text { el juego de roles. }\end{array}$ \\
\hline Desenvolvimiento & $\begin{array}{l}\text { Los participantes } \\
\text { muestran dominio } \\
\text { del tema en } \\
\text { sus diálogos } \\
\text { y evidencian } \\
\text { una adecuada } \\
\text { interpretación del } \\
\text { personaje en todo } \\
\text { momento. }\end{array}$ & $\begin{array}{l}\text { Los participantes } \\
\text { muestran dominio } \\
\text { del tema en } \\
\text { sus diálogos } \\
\text { y evidencian } \\
\text { una adecuada } \\
\text { interpretación del } \\
\text { personaje en la } \\
\text { mayor parte del } \\
\text { tiempo. }\end{array}$ & $\begin{array}{l}\text { Los participantes } \\
\text { muestran escaso } \\
\text { dominio del } \\
\text { tema en sus } \\
\text { diálogos y } \\
\text { evidencian una } \\
\text { inadecuada } \\
\text { interpretación } \\
\text { del personaje en } \\
\text { la mayor parte } \\
\text { del tiempo. } \\
\end{array}$ & $\begin{array}{l}\text { Los participantes } \\
\text { no muestran } \\
\text { dominio del tema } \\
\text { en sus diálogos, } \\
\text { ni evidencian } \\
\text { una adecuada } \\
\text { interpretación } \\
\text { del personaje. El } \\
\text { juego de rol está } \\
\text { completamente } \\
\text { improvisado. }\end{array}$ \\
\hline
\end{tabular}

Fuente. Elaboración propia.

\subsection{TIPO Y DISEÑO DEL ESTUDIO}

Es una investigación exploratoria de corte cualitativo basada en un estudio de casos y en un diseño transeccional. La investigación es exploratoria en cuanto se pretende dar una visión general de tipo aproximativo respecto al uso del juego de roles en los cursos consignados. Es un estudio de casos (Coller, 2005; Simons, 2011) porque respondió a la necesidad de evaluar, en forma sistemática, la información obtenida de la experiencia, pues su propósito fue evidenciar la evolución del desempeño académico de los y las estudiantes, tanto en los dos talleres de inducción como en la ejecución de la actividad. El diseño es transeccional debido a que se recolectan datos de la propuesta de forma discreta en el primer semestre de 2019.

La investigación se enmarca en el paradigma crítico (Melero, 2012), cuyos principios se dirigen hacia la transformación social y la democratización del currículum. Desde esta perspectiva, este estudio procura contribuir a contrarrestar el predominio de la clase tradicional de Historia, evidenciando la eficacia de las metodologías activas, en este caso del juego de roles, para promover y evaluar aprendizajes pedagógicos y disciplinares significativos.

En cuanto al análisis de resultados, cabe destacar que el objetivo de esta investigación es evaluar el juego de roles implementado en los cursos de la muestra. Para ello, la información obtenida de los instrumentos consignados es organizada y codificada mediante el programa Atlas.ti, versión 8.1; igualmente, se describen y relacionan diversas fuentes de datos a través de una doble triangulación, pues se recurre a la multimetódica y a la de múltiples fuentes.

La multimetódica (Blasco y Pérez, 2007) permite desarrollar un acercamiento hermenéutico y naturalista al contexto de estudio para interpretar los fenómenos en base a los significados que los sujetos participantes les otorgan. Por su parte, la triangulación de 
múltiples fuentes, como sugieren Delgado y Estepa (2016), ayuda a superar las debilidades teóricas y analíticas que podrían darse ante la recogida de información individual. De este modo, mientras en la primera triangulación se lleva a cabo un análisis interpretativo para destacar las convergencias y divergencias existentes entre las concepciones de los estudiantes y las del investigador; en la segunda, se refuerza la validez y la fiabilidad de dichas interpretaciones con apoyo de los datos recogidos en la planilla de asistencia y en la rúbrica de evaluación. Siguiendo esta doble triangulación, se ejecuta un análisis documental de todos los insumos de información para delimitar, en forma inductiva, la categoría y subcategorías de análisis que orientaron la evaluación del juego de roles (tabla 5).

Tabla 5. Matriz de categoría y subcategorías analizadas

\begin{tabular}{|c|c|}
\hline Categoría & Subcategorías \\
\hline $\begin{array}{l}\text { Evaluación de la } \\
\text { experiencia }\end{array}$ & $\begin{array}{l}\text { - Familiarización del profesorado en formación con el juego de roles } \\
\text { como estrategia didáctica y evaluativa } \\
\text { - } \quad \text { Dominio de las habilidades propias del pensamiento histórico } \\
\text { - } \text { Interrelación entre los integrantes de los equipos de trabajo } \\
\text { - Asistencia y desempeño académico del alumnado durante el } \\
\text { desarrollo de la propuesta } \\
\text { - Valoración de la experiencia por parte del estudiante } \\
\text { - Limitaciones de la actividad implementada } \\
\text { - Aspectos claves de la estrategia docente que se deben resguardar en } \\
\text { futuras intervenciones }\end{array}$ \\
\hline
\end{tabular}

Fuente. Elaboración propia

Como señalan Gómez y Miralles (2015), la evaluación de los aspectos consignados responde a las particularidades epistemológicas, pedagógicas y cognitivas de la disciplina histórica y su respectiva enseñabilidad, y busca analizar los resultados de una experiencia educativa compleja, que tiene como propósito mejorar los aprendizajes pedagógicos e históricos de los sujetos participantes.

\section{EVALUACIÓN DE LOS APRENDIZAJES OBTENIDOS A TRAVÉS DEL JUEGO DE ROLES}

Una primera cuestión que se evidencia de esta experiencia es la escasa familiarización que tienen los sujetos participantes con el juego de roles como estrategia didáctica y evaluativa. Esto se ve reflejado en la reticencia con la que reaccionaron tres alumnos del curso "Conociendo Chile de los siglos XIX y XX" cuando se les entregó el instructivo de la actividad, a pesar de que durante las tres semanas previas a la misma se ejecutaron dos talleres de inducción. Como destaca el registro de una estudiante de dicha asignatura, las "reticencias observadas se explican por el desagrado a actuar o expresarse en público" (P13), lo que, según Antony (2012), tiende a estar vinculado con la baja autoestima y el temor a la ridiculez de parte del alumnado. Este problema, como precisa otra estudiante 
en su reporte, "puede entorpecer el trabajo en equipo y comprometer el posterior éxito de la actividad" (P18). Al respecto, una alumna del mismo curso señala que este tipo de inconveniente "podría replicarse con nuestros futuros estudiantes, quienes pueden sentir rechazo o vergüenza para realizar el juego de roles frente a sus compañeros" (P25).

Para evitar esta dificultad, el itinerario de trabajo hubiese podido contemplar la posibilidad de grabar la representación y entregarla en un soporte físico, ya sea en un CD o pendrive. Sin embargo, no se optó por esta modalidad porque uno de los objetivos basales de la propuesta era potenciar las habilidades interpersonales de los futuros docentes, tales como el trabajo en equipo, la responsabilidad, la flexibilidad y, muy especialmente, la capacidad de oratoria. Por tal motivo, resulta indispensable que el profesorado en formación pueda vencer sus posibles miedos para comunicarse ante una audiencia, pues las aulas del siglo XXI, como destacan Romero y Hurtado (2017), requieren de docentes seguros, decididos, expresivos y cercanos, y que sepan promover aprendizajes significativos en sus alumnos a través de metodologías activas de enseñanza.

Otra de las limitaciones observadas es la gran carga de trabajo que implicó el juego de roles para el alumnado y el docente. Para los futuros profesores, el desarrollo de esta actividad generó frustración y aburrimiento en algunos momentos debido a que requirió bastante tiempo y dedicación para realizar las tareas consignadas. En cuanto al docente, el diseño, implementación y evaluación de la experiencia fue una labor compleja y muy costosa en tiempo; por esta razón, es fundamental impulsar la creación de equipos docentes que tengan la misión de planificar actividades centradas en estrategias constructivistas e innovadoras.

A pesar de las dificultades descritas, la mayoría de los y las estudiantes considera que la actividad del juego de roles es significativa y motivadora para formar el pensamiento histórico y potenciar las habilidades blandas.

En cuanto al desarrollo del pensamiento histórico, la propuesta permitió a los futuros docentes comprender y trabajar directamente con los principales conceptos (fuentes históricas, causas y consecuencias, tiempo histórico, relevancia histórica y empatía histórica) que conforman este metaconcepto.

Uno de los aprendizajes de segundo orden que los y las estudiantes lograron dominar con mayor facilidad fue la empatía histórica, ya que el laboratorio histórico realizado para investigar sobre el personaje asignado contribuyó no solo para que fuesen capaces de ponerse en el "lugar" y en el contexto histórico de este, sino también para elaborar los diálogos, el vestuario y la escenografía requerida en el juego de roles. Por tal motivo, según una estudiante de "Ciencias Sociales II", esta actividad permitió desarrollar "la imaginación o pensamiento histórico para repensar o reconstruir la historia desde el rigor que exige la disciplina" (P4).

Al respecto, cabe destacar que "sin imaginación no se puede escribir historia", tal como lo expresa Tenorio (2012), puesto que el historiador también usa este recurso para dar coherencia a su relato dentro de unos criterios científicos propios de la especialidad. Por esta razón, hay que tener en cuenta que el alumnado no sólo puede aprender desde la realidad concreta, sino también desde aquella que es intangible o abstracta. En este contexto, la empatía histórica ofrece al estudiante la posibilidad de reflexionar sobre las situaciones históricas representadas y entender las posibles intenciones o motivaciones de vida de los personajes interpretados. Esto, desde la perspectiva docente, ayuda a los futuros profesores a no caer en la lógica presentista a la hora de analizar la temática tratada. 
En el caso contrario, la habilidad del pensamiento histórico donde los y las estudiantes presentaron mayores dificultades para trabajar fue el análisis de fuentes porque, si bien fueron capaces de desarrollar un laboratorio histórico, no pudieron comprender y simular correctamente el procedimiento de las cuatro heurísticas (heurística de origen, contextualización, lectura cerrada y corroboración heurística) que utiliza el historiador para crear sus narrativas históricas. A esto se le debe sumar que la escasa información que existe sobre los personajes representados y la inexperiencia del alumnado para consultar bases de datos de revistas de alto impacto y de archivos digitales, como el sitio de memoria chilena y el portal de archivos españoles, dificultaron el trabajo basado en competencias históricas; sin embargo, el continuo proceso de acompañamiento docente pudo atenuar estas complicaciones. Esta situación problemática se explica porque es el primer acercamiento que los cursos de la muestra tienen con una experiencia de innovación de este tipo, por lo que es imperativo seguir desarrollándolas para que los futuros profesores, a través de ejercicios prácticos de indagación, puedan enseñar a las nuevas generaciones de estudiantes a ejecutar un riguroso análisis descriptivo y heurístico de fuentes para investigar un problema histórico específico.

Aunque el alumnado tuvo que enfrentar los problemas enunciados dentro de la fase de análisis de fuentes, el trabajo investigativo consignado para preparar el juego de roles permitió a los futuros profesores evidenciar el marcado carácter eurocéntrico y androcéntrico de la historia oficial que se concibe en el currículum escolar. Partiendo de la base de que la mayor parte de los personajes históricos interpretados eran hombres europeos, el estudiantado coincide en la necesidad de reivindicar el papel de la mujer como un sujeto histórico que ha aportado de forma relevante al desarrollo de la humanidad; asimismo, también plantea la urgencia de analizar y estudiar miradas historiográficas extraeuropeas para entender la Historia como una disciplina cuyos límites interpretativos van mucho más allá de la perspectiva eurocéntrica o de la sesgada visión del hombre blanco y adulto.

La necesidad de repensar la historia oficial, a través del uso de metodologías activas, es cada vez más latente debido a que la investigación reciente ha constatado la invisibilización de las mujeres y de los actores no europeos en los discursos hegemónicos de la historia (Ramallo, 2014; Ortega y Pagès, 2018; Marolla y Saavedra, 2020). La ausencia de tales sujetos históricos, evidenciada en la adjudicación de arquetipos definidos e integrados en el imaginario colectivo, es un hecho reconocible tanto en las programaciones curriculares como en las prácticas del profesorado.

En esta línea, el juego de roles ayudó a los docentes en formación a trabajar con interpretaciones en lugar de certezas porque fue una instancia enriquecedora para comprender, desde una mirada crítica, la historia que se enseña en el aula. Por tanto, y siguiendo los planteamientos del pensamiento histórico, no se trata de que los sujetos participantes se formen como historiadores, sino que, a través de la comprensión epistemológica de la disciplina, sean capaces de valorar las diferentes argumentaciones o afirmaciones que se hacen del pasado para describir a las sociedades presentes.

Desde un punto de vista didáctico, es importante resaltar que el trabajo basado en competencias históricas contribuyó a que el estudiantado obtuviera los aprendizajes teóricos necesarios para la implementación del juego de roles, puesto que el protocolo de la actividad y la experiencia de los dos talleres de inducción fueron insumos indispensables tanto para alcanzar la correcta ejecución de esta estrategia como para el logro de los 
múltiples formatos de las propuestas presentadas (entrevistas simuladas, personificaciones, dramatizaciones, entre otras). Por tal motivo, el saber hacer del juego de roles fue la fase mejor lograda y más valorada por el alumnado, ya que, aparte de estudiarla como un concepto, tuvieron la posibilidad de aprender su metodología en la praxis.

De esta manera, este tipo de innovación contribuye a que los futuros profesores adquieran la metodología de la investigación histórica para que la puedan transferir al área pedagógica. Para lograr este objetivo, y siguiendo a Rivera y Mondaca (2013),

Es de vital importancia el rol docente, a través del currículum oculto que oralmente trasmitimos y que debe ser inspirado por un compromiso social que recupere el papel de sujeto reflexivo y transformador de la Historia, activando la pereza e incluso el miedo de la Comodidad Neutra (el Pacifismo social), que aparece en muchos docentes al abordar metodológicamente el estudio de la historia reciente desde la óptica descriptiva y mínimamente interpretativa (pp. 399-400).

Respecto al dominio de habilidades interpersonales, la alta adhesión a la actividad se traduce en la presentación de propuestas originales por parte de los alumnos, quienes demostraron, en términos generales, una gran dedicación, creatividad y flexibilidad para desenvolverse en una estrategia didáctica y evaluativa escasamente utilizada en la universidad. Por ejemplo, destaca la representación de Thomas Cochrane realizada por dos estudiantes de la asignatura "Conociendo Chile de los siglos XIX y XX". En ella, interpretaron al experimentado marino inglés como un personaje que salía de una botella para explicar los ejes más importantes de su vida y que, principalmente, deseaba aclarar que no solo era el nombre de calles famosas del país.

El alumnado, además, valora el trabajo colaborativo que posibilitó esta estrategia. Como destaca una estudiante de "Ciencias Sociales II", "cada equipo tuvo que liderar un diálogo fructífero porque nos vimos enfrentados a la necesidad de establecer negociaciones y acuerdos para preparar la actividad" (P3). Otra alumna de la misma asignatura añade que "gracias al trabajo realizado, cada una pudo reconocer las fortalezas y dificultades de su compañera al momento de realizar el juego de roles" (P2). No obstante, también se observaron diversos inconvenientes que repercutieron en el trabajo en equipo, tales como la escasa iniciativa de cinco estudiantes para realizar las tareas y la falta de interés de cuatro participantes por investigar sobre la temática representada.

Del mismo modo, cabe mencionar que la mayor parte de los y las estudiantes coinciden en que se sienten mejor preparados/as para enfrentar el mercado laboral después de desarrollar esta experiencia. Esto se explica por la motivación generalizada que provocó el juego de rol en los profesores en formación, quienes, en su futura labor, tienen el gran reto de enseñar la disciplina histórica mediante la implementación de estrategias didácticas y evaluativas innovadoras.

Esta valoración positiva se explica porque el juego de roles, además de los aspectos ya mencionados, permitió mejorar la disposición y la participación del alumnado en el aula. De hecho, a medida que los estudiantes interpretaban sus respectivos personajes, se volvían cada vez más espontáneos y entusiastas. Sin embargo, cabe notar que cuando algunos de ellos hicieron su representación pública experimentaron cierto pánico escénico, el cual fue oportunamente identificado por el docente para ayudarles a superar esta dificultad mediante un feedback, que se basó en propiciar un clima de confianza y respeto. Como 
resultado de esta acción pedagógica que buscaba minimizar los niveles de estrés y ansiedad de las evaluaciones tradicionales, los juegos de roles mejoraron clase a clase, tal como se evidencia en las calificaciones (tabla 6 y 7) y en el cuaderno de campo de los y las estudiantes.

En la experiencia se destaca también el alto grado de compromiso y responsabilidad del estudiantado, ya que todos y todas cumplieron con el desarrollo de la actividad. De igual forma, también se aprecia en que el 92,5\% de los participantes asistieron regularmente a las clases que contemplaron la preparación y presentación del juego de roles. Los casos de inasistencia son puntuales y cuentan con el justificativo correspondiente.

En cuanto al desempeño académico logrado con el juego de roles, resalta el $100 \%$ de aprobación por parte del alumnado, evidenciándose una mejora importante en los resultados obtenidos en una evaluación tradicional con los mismos contenidos, donde el porcentaje de aprobación eran cercanos al $85 \%$ para el caso de las asignaturas donde se implementó la experiencia.

Si bien la evaluación solo contempló la calificación final como oficial, es importante mencionar que también hubo dos notas "simbólicas" correspondientes a cada taller de inducción, tal como se aprecia en las tablas 6 y 7. Dichas calificaciones sirvieron para constatar semanalmente la mejora generalizada y progresiva del estudiantado y la eficacia del proceso de retroalimentación entregado. Debido a que la mayoría de los alumnos acogieron las sugerencias del profesor para mejorar sus respectivos juegos de roles, se obtuvieron resultados positivos que se ven reflejados en que todas las calificaciones superan el 5,0, en una escala de 1,0 a 7,0. De esta manera, se observa un desempeño similar en ambos cursos, a pesar de que cada uno de estos sigue un itinerario formativo diferenciado por las menciones que existen en la carrera de Pedagogía en Educación Básica.

Tabla 6. Registro de calificaciones del juego de roles en la asignatura "Ciencias Sociales II"

\begin{tabular}{|c|c|c|c|}
\hline Equipo de estudiantes & Taller de inducción 1 & Taller de inducción 2 & Juego de roles \\
\hline P1+P2+P3 & 5,4 & 5,8 & 5,8 \\
\hline P4+P5 & 5,8 & 5,8 & 6,1 \\
\hline P6+P7 & 6,1 & 6,4 & 7,0 \\
\hline P8+P9 & 5,8 & 6,1 & 6,4 \\
\hline
\end{tabular}

Fuente. Elaboración propia. 
Tabla 7. Registro de calificaciones del juego de roles en la asignatura "Conociendo Chile de los siglos XIX y XX"

\begin{tabular}{|c|c|c|c|}
\hline Equipo de trabajo & Taller de inducción 1 & Taller de inducción 2 & Juego de roles \\
\hline P10+P11 & Inasistencia & 5,8 & 5,8 \\
\hline P12+P13 & 6,1 & Inasistencia & 6,4 \\
\hline P14+P15 & 6,1 & 6,1 & 6,7 \\
\hline P16+P17 & 6,4 & 6,4 & 7,0 \\
\hline P18+P19 & 6,7 & 6,7 & 7,0 \\
\hline P20+P21 & 5,8 & 5,8 & 6,4 \\
\hline P22+P23 & 5,4 & 5,8 & 6,1 \\
\hline P24+P25 & 6,7 & Inasistencia & 7,0 \\
\hline P26+P27 & Inasistencia & 6,4 & 6,7 \\
\hline
\end{tabular}

Fuente. Elaboración propia

Asimismo, resulta conveniente precisar que la actividad ha reforzado la buena relación entre el docente y los estudiantes, porque se convirtió en un espacio de mayor interacción entre las partes. De hecho, los y las participantes reconocen y valoran el proceso de evaluación formativa realizado a lo largo del trabajo, lo cual ha contribuido significativamente a mejorar el clima de aula donde se forjan los aprendizajes.

También es indispensable resaltar que el profesorado en formación justifica la necesidad de replicar y adaptar la estrategia del juego de roles al contexto de sus futuros alumnos y alumnas, ya que no la han utilizado en otra asignatura o aplicado en sus prácticas pedagógicas progresivas.

En el ámbito previsto, tres estudiantes de "Conociendo Chile de los siglos XIX y XX" y dos de "Ciencias Sociales II" sostienen en que se les insiste de manera reiterada en que deben usar metodologías activas en su futuro desempeño profesional; no obstante, añaden que son escasos los esfuerzos docentes para enseñar, en forma práctica, su proceso de implementación. En esta línea, argumentan que la formación inicial del profesorado se focaliza fundamentalmente en la disciplina histórica porque, según la percepción de los alumnos, el saber didáctico y evaluativo de la especialidad se relega, en forma recurrente, a un segundo plano.

Sin embargo, antes de que los docentes en formación puedan replicar la propuesta en el aula con sus futuros estudiantes, deben tener en cuenta su nivel escolar, sus aprendizajes previos y las especificidades del contexto educativo. Al respecto, cabe señalar que los participantes están conscientes de estas condicionantes, por lo que la probabilidad de que apliquen la propuesta en su futura labor es bastante elevada.

Pese a lo anterior, la mayoría argumenta que el juego de roles solo es apto para trabajar con estudiantes de 10 años hacia delante, porque en los/as niños/as más pequeños/as puede generar posibles problemas de entendimiento acerca del instructivo de trabajo o del personaje histórico que deseen representar debido a que no cuentan con la madurez cognitiva 
necesaria. Adicionalmente, coinciden en que desde su rol como futuro/a profesor/a deben mantener una postura firme y clara frente a sus estudiantes para fomentar un ambiente de respeto que propicie el adecuado desarrollo de la actividad; y añaden, además, en que se debe supervisar constantemente la información investigada por los alumnos para evitar errores o que centren la indagación en fuentes poco confiables.

Por último, a la luz de la experiencia implementada, resulta relevante considerar las dificultades observadas y resguardar los siguientes aspectos en futuras intervenciones: la sobrecarga académica que implica para el docente y el alumnado; el poco interés de algunos estudiantes por la temática y/o la estrategia trabajada; el posible pánico escénico; el escaso conocimiento que existe sobre la mayoría de los temas abordados; y la inexperiencia de los futuros docentes para consultar bases de datos digitales que les permita acceder a fuentes primarias y secundarias especializadas. Se está plenamente consciente de estos problemas, por lo que, desde la perspectiva docente, requerirá un mayor esfuerzo para trabajar de forma más frecuente en propuestas similares, donde el profesorado en formación pueda potenciar su pensamiento histórico y mejorar sus habilidades sociales.

\section{CONCLUSIONES}

Los resultados obtenidos en esta investigación demuestran que el juego de rol no es simplemente una estrategia con fines lúdicos y motivacionales, sino que también sirve para evaluar y promover de forma eficaz el desarrollo de aprendizajes significativos en los cursos analizados, pues se pudo apreciar, por una parte, una mejora constante y generalizada en el desempeño académico de los y las estudiantes; y por otra, se comprobó que fueron capaces de liderar un proceso de aprendizaje construido y relacionado con sus conocimientos previos. En este ámbito, cabe destacar que la evaluación del juego de roles privilegió el proceso formativo por sobre el sumativo, pues no se limitó a la dimensión cuantitativa y puso énfasis en el desarrollo de habilidades propias del pensamiento histórico que trascienden la memorización o retención de aprendizajes de primer orden como fechas, personajes y grandes hitos de la historia.

Para el profesorado en formación, esta propuesta fue un espacio valioso para relacionar la teoría con la praxis, situación que, según los propios alumnos, debería replicarse en las demás asignaturas de su carrera. Para el docente, esta experiencia permitió reflexionar sobre la propia práctica pedagógica, ya que la investigación realizada contribuyó a la mejora de los aprendizajes de los participantes y a la comprensión de las especificidades evaluativas y didácticas de la disciplina histórica.

Estos resultados son coherentes con los obtenidos en otros contextos educativos similares (Carbó y Pérez, 2010; Beidatsch y Broomhall, 2010), y confirman la trascendencia e imperiosa necesidad de incorporar estrategias didácticas y evaluativas innovadoras en la clase de Historia porque resulta indispensable contrarrestar el predominio de las clases magistrales, ya que no son capaces de promover el pensamiento histórico y el desarrollo de habilidades blandas.

Esta investigación no está exenta de limitaciones. La experiencia se realizó en una muestra pequeña seleccionada de manera intencional. La evaluación de aprendizajes pedagógicos e históricos significativos es una labor muy compleja, puesto que los resultados obtenidos dependen principalmente de las características del grupo-curso y de su madurez 
para realizar las tareas asignadas; igualmente, obedecen a las particularidades epistémicas, pedagógicas y cognitivas de la disciplina en que se inserta la propuesta, pues, como se evidenció, este factor es determinante a la hora de planificar una actividad como el juego de roles previsto. Por lo tanto, no se garantizan los resultados de esta intervención para otros casos, ya que cada uno es único y presenta sus propios problemas y ventajas.

Asimismo, dadas las dificultades y los aspectos que se deben cautelar en futuras clases, hay que tener en cuenta que los juegos de roles no son la panacea y desde luego no se propone que sustituya totalmente al modo de enseñanza tradicional. No obstante, al menos podría considerarse como un método didáctico-evaluativo adicional y complementario que puede ser usado en parte del tiempo completo destinado a una asignatura, o como apoyo de los trabajos de investigación que deban realizar los alumnos en la misma.

De acuerdo a esto último, es importante destacar que el juego de roles puede tener un potencial impacto en la comunidad educativa, pues constituye una interesante propuesta de innovación que integra distintas metodologías didácticas vivenciales y de evaluación situada (el juego de roles, el método de casos y la evaluación formativa) que están en consonancia con los modelos pedagógicos centrados en el alumno.

El análisis de esta experiencia se presenta como un sustento teórico y empírico sólido para continuar desarrollando nuevas investigaciones y propuestas pedagógicas en el aula. Desde el punto de vista investigativo, se deberían realizar estudios a futuro que complementen los resultados obtenidos sobre el juego de roles implementado para promover la formación de competencias históricas y de habilidades interpersonales, o bien emprender investigaciones sobre nuevos casos donde se desarrolle el juego de roles u otras estrategias innovadoras, como el debate, el aprendizaje basado en problemas y el puzle, que tengan los mismos fines formativos. En cuanto al aspecto empírico, la propuesta ofrece referentes metodológicos para ejecutar la experiencia en diversos cursos de Educación Básica, Media y Superior que tengan como propósito el aprendizaje de la Historia, o bien en asignaturas donde se impartan otras disciplinas, siempre y cuando se tomen en cuenta las características del contexto educativo y las particularidades de la especialidad en el diseño de la intervención.

\section{REFERENCIAS BIBLIOGRÁFICAS}

Álvarez, H. (2020). El uso del debate en la Educación en Derechos Humanos. Problemas, desafíos y potencialidades. Mendive, 18(2), 219-234.

Antony, M. (2012). Como superar la timidez y el miedo a hablar en público. Barcelona: Editorial Amat.

Armas, J., Moreira, A., Maia, C. y Conde, J. (2019). La historia de Portugal en las aulas de Educación Básica. ¿Formar patriotas o educar ciudadanos? Revista Electrónica Interuniversitaria de Formación del Profesorado, 22(2), 67-80. Doi: https://doi.org/10.6018/reifop.22.2.369521

Ausubel, D. (1976). Significado y aprendizaje significativo. En D. Ausubel, J. Novak y H. Hanesian (Eds.), Psicología educativa: Un punto de vista cognoscitivo (pp. 53-106). México: Trillas.

Beidatsch, C. y Broomhall, S. (2010). Is this the past? The place of role-play exercises in undergraduate history teaching. Journal of University Teaching \& Learning Practice, 7(1), 1-22.

Blasco, J. y Pérez, J. (2007). Metodologías de investigación en las ciencias de la actividad física y el deporte: Ampliando horizontes. Alicante: Club Universitario.

Carbó, J. y Pérez, P. (2010). Fuentes históricas de los juegos de rol: Un experimento para la Didáctica de la Historia Antigua. Teoría de la Educación. Revista Interuniversitaria, 11(3), 149-167. 
Chapman, A. (2011). Historical interpretations. In I. Davies (Ed.), Debates in history teaching (pp. 96-109). Oxon: Routledge.

Coller, X. (2005). Estudio de casos. Madrid: Centro de Investigaciones Sociológicas.

Craciun D. (2010). Role-playing as a creative method in science education. Journal of Science and Arts, 1(12), 175-182.

Delgado, E. y Estepa, J. (2016). Ciudadanía y memoria histórica en la enseñanza de la historia: Análisis de la metodología didáctica en un estudio de caso en ESO. Revista de Investigación Educativa, 34(2), 521-534.

Domínguez, J. (2015). Pensamiento histórico y evaluación de competencias. Barcelona: Editorial Graó.

Evans, E. (2010). Orientaciones metodológicas para la investigación-acción. Lima: Ministerio de Educación.

Fernández, J., Prieto, E., Alcaraz, V., Sánchez, A. y Grimaldi, J. (2018). Aprendizajes significativos mediante la gamificación a partir del juego de rol: "Las Aldeas de la Historia". Espiral. Cuaderno del Profesorado, 11(22), 69-78.

Gaete, R. (2011). El juego de roles como estrategia de evaluación de aprendizajes universitarios. Educación y Educadores, 14(2), 289-307.

Gatica. F. y Uribarren, T. (2013). ¿Cómo elaborar una rúbrica? Investigación en Educación Médica, 2(1), 61-65.

Gómez, M. (2006). Introducción a la metodología de la investigación científica. Córdoba: Brujas.

Gómez, C., Ortuño, J. y Molina, S. (2014). Aprender a pensar históricamente. Retos para la historia en el siglo XXI. Tempo e Argumento, 6(11), 5-27.

Gómez, G. y Miralles, P. (2015). ¿Pensar históricamente o memorizar el pasado? La evaluación de los contenidos históricos en la educación obligatoria en España. Revista de Estudios Sociales, (52), 52-68.

Gómez, C., López, R. y Prats, J. (2017). Las competencias históricas en el horizonte. Propuestas presentes y perspectivas de futuro. En C. Gómez, P. Miralles, R. López y J. Prats (Coords.), Enseñanza de la historia y competencias educativas (pp. 191-206). Barcelona: Editorial Graó.

Gómez, C., Miralles, P., López, R. y Prats, J. (2017). Enseñanza de la historia y competencias educativas. Barcelona: Editorial Graó.

Gómez, C., Miralles, P. y Chapman, A. (2017). Los procedimientos de evaluación en la clase de Historia. Un análisis comparativo a través de las opiniones de los docentes en formación en España e Inglaterra. Revista Electrónica Interuniversitaria de Formación del Profesorado, 20(2), 45-61.

Gómez, C. y Chapman, A. (2017). Enfoques historiográficos y representaciones sociales en los libros de texto. Un estudio comparativo, España-Francia-Inglaterra. Historia y Memoria de la Educación, (6), 319-361.

Gómez, C., Rodríguez, R. y Mirete, A. (2018). Percepción de la enseñanza de la historia y concepciones epistemológicas. Una investigación con futuros maestros. Revista Complutense de Educación, 29(1), 237-250.

Gómez, C., Ortuño, J. y Miralles, P. (2018). Enseñar ciencias sociales con métodos activos de aprendizaje. Reflexiones y propuestas a través de la indagación. Barcelona: Editorial Octaedro.

Grande de Prado, M. y Abella, V. (2010). Los juegos de rol en el aula. Teoría de la Educación. Educación y Cultura en la Sociedad de la Información, 11(3), 56-84.

Hernández, R., Fernández, C. y Baptista P. (2014). Metodología de la investigación. México: McGraw-Hill Interamericana.

Itzkovich, Y. (2019). The connection between perceived constructivist learning environments and faculty uncivil authoritarian behaviors. Higher Education, 77(3), 437-454.

Marolla, J. y Saavedra, C. (2020). Presencia y ausencia de América Latina. La didáctica de las ciencias sociales y la interculturalidad como desafío para la enseñanza. Revista Interdisciplinaria de Estudios Latinoamericanos, 4(1) 75-92. 
Melero, N. (2012). El paradigma crítico y los aportes de la investigación acción participativa en la transformación social: Un análisis desde las Ciencias Sociales. Cuestiones Pedagógicas, 21, 339-355.

Molina, S. y Ortuño, J. (2018). Concepciones del profesorado iberoamericano de Secundaria sobre la contribución del patrimonio local al desarrollo del pensamiento histórico. Estudios Pedagógicos, 43(4), 185-202. Doi:10.4067/S0718-07052017000400010.

Monereo, C. y Domínguez, C. (2014). La identidad docente de los profesores universitarios competentes. Educación XX1, 17(2), 83-104.

Murphy, J. (2011). Más de 100 ideas para enseñar historia. Barcelona: Editorial Graó.

López, M. (2020). Concepciones y prácticas de profesores de Historia sobre la construcción didáctica del tiempo histórico mediado por el uso de las TIC. Cuaderno de Pedagogía Universitaria, 17, 29-43. Doi: https://doi.org/10.29197/cpu.v17i33.372

Ortega, D. y Pagès, J. (2018). Género y formación del profesorado: Análisis de las guías docentes del área de Didáctica de las Ciencias Sociales. Contextos Educativos. Revista de Educación, (21), 53-66.

Palacios, N., Chaves, L. y Martin, W. (2020). Desarrollo del pensamiento histórico. Análisis de exámenes de los estudiantes. Magis. Revista Internacional de Investigación en Educación, (13), 1-29. Doi: 10.11144/Javeriana.m13.dpha

Prats, J. (2011). Didáctica de la Geografía y la Historia. Barcelona: Editorial Graó. . (2016). Combates por la historia en educación. Enseñanza de las Ciencias Sociales. Revista de Investigación, (15), 145-153.

Quezada, G., Castro, M., Oliva, J., Gallo, C. y Quezada, M. (2020). Juego de roles para identificar la violencia familiar en universidades, Piura - Perú. Revista Espacios, 41(20), 51-60.

Ramallo, F. (2014). Enseñanzas de la historia y lecturas descoloniales: Entrecruzamientos hacia los saberes de otros mundos posibles. Revista Entramados - Educación y Sociedad, 1(1), 43-59.

Rivera, P. y Mondaca, C. (2013). El aporte de la enseñanza de la historia reciente en Chile: Disensos y consensos desde la transición política al siglo XXI. Estudios Pedagógicos, 39(1), 393-401. Doi:10.4067/S0718-07052013000100023

Romera, C., López, A. y Monteagudo, J. (2019). Desarrollo del pensamiento histórico en las aulas a través de un programa formativo para Enseñanza Secundaria. Revista Electrónica Interuniversitaria de Formación del Profesorado, 22(2), 81-93. Doi: https://doi.org/10.6018/ reifop.22.2.363911

Romero, A. y Hurtado, S. (2017). ¿Hacia dónde va el rol del docente en el siglo XXI? Estudio comparativo de casos reales basados en las teorías constructivista y conectivista. Hekademos, (22), 84-92.

Sabino, C. (2014). El proceso de investigación. Guatemala: Episteme.

Salazar, R., Orellana, C., Muñoz, C. y Bellati, I. (2017). El aula como laboratorio histórico: la Guerra Civil Española. En C. Gómez, P. Miralles, R. López y J. Prats (Coords.), Enseñanza de la historia y competencias educativas (pp. 110-126). Barcelona: Editorial Graó.

Seixas, P. y Morton, T. (2012). The big six historical thinking concepts. Toronto: Nelson.

Simons, H. (2011). Estudio de caso: Teoría y práctica. Madrid: Morata.

Suárez, X., Castro, N. y Muñoz, C. (2020). Uso de juego de roles con grabación de video para el desarrollo de la competencia de entrevistar en estudiantes de psicología. Revista Electrónica Educare, 24(2), 1-18. Doi: https://doi.org/10.15359/ree.24-2.2

Tarr, R. (2018). A history teaching toolbox. Practical classroom strategies. London: CreateSpace Independent Publishing Platform.

Tenorio, M. (2012). Culturas y memoria: Manual para ser historiador. Barcelona: Tusquets Editores.

Van Boxtel, C., Van Drie, J. y Stoel, G. (2020). Improving teachers' proficiency in teaching historical thinking. En The Palgrave Handbook of History and Social Studies Education (pp. 97-117). Springer International Publishing. Doi: https://doi.org/10.1007/978-3-030-37210-1_5 
Estudios Pedagógicos XLVI N²: 97-121, 2020 PROMOVIENDO APRENDIZAJES SIGNIFICATIVOS EN LA ENSEÑANZA UNIVERSITARIA DE LA HISTORIA A TRAVÉS DE UN JUEGO DE ROLES

Vygotsky, L. (1989). El desarrollo de los procesos psicológicos superiores. Barcelona: Editorial Crítica.

Wenner, M. (2011). La importancia de jugar. Mente y Cerebro, (46), 38-45.

Young, S. y Shaw, D. (2014). Profiles of effective college and university teachers. The Journal of Higher Education, 70(6), 670-686. 
